\title{
An information integration approach to phenomenal causality
}

\author{
ANNE SCHLOTTMANN \\ University College, London, England \\ and \\ NORMAN H. ANDERSON \\ University of California, San Diego, California
}

\begin{abstract}
Phenomenal causality was studied by using Michotte's launch event, in which successive motion of two objects evokes an immediate perception that the first motion causes the second. Information integration theory was used to address the complementary issues of invariant perceptual structure and individual differences in phenomenal causality. Three informational cues were varied conjointly: temporal and spatial contiguity of the two motions, and the ratio of their speeds. The dependent measure was a judgment of degree of causality or naturalness. The results showed that individual differences were related to these instruction conditions; the subjects showed five distinctive response patterns. Two were the modal patterns elicited by the instructions, and the others fell in between. The averaging model gave a good account of the data, with meaningful parameter estimates. Individual differences were localized in cue evaluation, whereas their integration into a unified judgment followed an invariant averaging rule. The results allow some reconciliation between Michotte and his critics.
\end{abstract}

Everyday cognition relies heavily on our recognizing causal relations among events. This article concerns phenomenal causality, as it was investigated by Albert Michotte (1946/1963). Michotte's phenomenological approach has evoked considerable criticism as well as conflicting results in subsequent work. Individual differences and their interpretation have been a central point of controversy.

The present study applies an information-integration approach to phenomenal causality. The basic concern is twofold-with the invariant structure of phenomenal causality, and with the individual difference controversy. These two concerns are complementary: Individual differences cannot be understood at a merely descriptive level; a theoretical model of the process in which they function is required as well. Accordingly, this study is focused on a fine-grained parametric analysis of multiple perceptual cues and on a quantitative model for the way in which these cues are integrated into a unitary causal impression. This model then provides a tool for analyzing individual differences in some depth.

\section{Michotte's Claims}

Michotte explored conditions in which people say they see one event causing or producing another event. A prime

This work was supported by Grant PHS HD22932 from the National Institute of Child Health and Development. We thank John Kruschke, Alan Leslie, and Peter White for valuable comments on a previous version, and Bill Gaver for programming support and discussion. Address correspondence to A. Schlottmann at the Department of Psychology, University College London, Gower Street, London WC1E 6BT, England. example is his well-known launch event, illustrated in the motion sequence of Figure 1. Most people see the first object, $A$, pushing (launching) the second object, $B$, thus producing its movement.

Michotte studied extensively the determinants of phenomenal causality, such as temporal and spatial contiguity at the point of impact, and speed of the objects $A$ and $B$. The optimal conditions for the causal impression, as Michotte stressed, depend on the particular configuration of stimulus parameters. At higher speeds, for example, larger disruptions in spatiotemporal contiguity are necessary if the causal impression is to be destroyed. Given a suitable balance in motion parameters, observers are said to immediately experience one event causing another (also see Michotte \& Thinès, 1963/1991).

Michotte considered such phenomenal causality to arise as a Gestalt property of specific motion sequences. Such a perceptual organization for causality could account for the ease that people seem to have in recognizing some causal relations. Indeed, even infants appear to react to the specifically causal structure of launch events (Leslie, 1982, 1984; Leslie \& Keeble, 1987; Oakes \& Cohen, 1990; also see White, 1988).

Michotte's claim had two aspects, one empirical, the other theoretical. The empirical claim was a demonstration of phenomenal causality in visual scenes devoid of actual causality. This is akin to Duncker's (1935/1945) famous example of a light going on at one end of a hallway at the moment a door is shut at the other end, which yields a causal illusion that runs counter to factual knowledge. Phenomenal causality may thus be considered a per- 


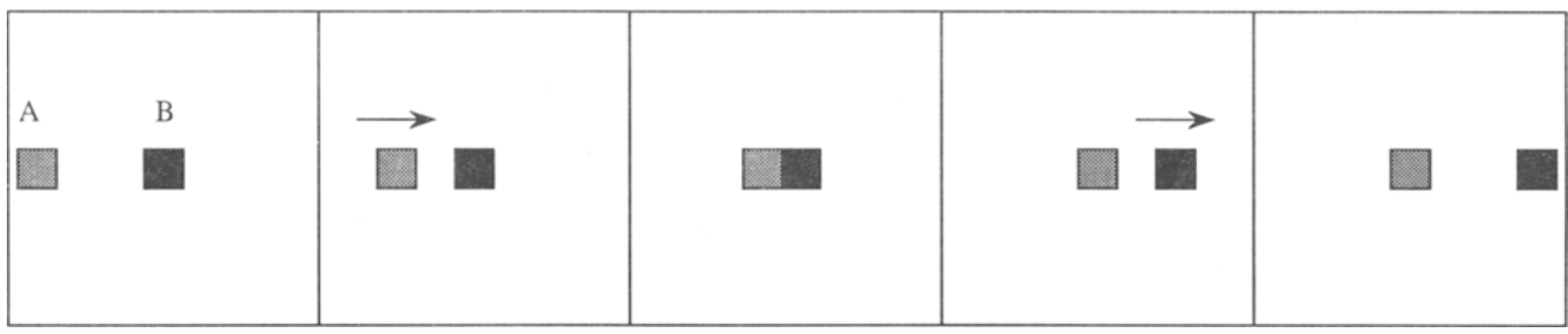

Figure 1. Prototypical launch event. Object $A$ moves toward object $B$, which is stationary. Immediately after collision, object $A$ is stationary and object $B$ moves away from it.

ceptual illusion, analogous to apparent movement. It should not be confused with the perceptual correlate of physical causation (see Runeson, 1977).

The main objection to this empirical claim is that not everyone experiences the Michotte effect (Beasley, 1968; Boyle, 1960; Gemelli \& Cappellini, 1958). Under supposedly optimal conditions, at least $10 \%$ of subjects report no causal perception, and many subjects shift between causal and noncausal reports. This matter was known to Michotte and was reported by Boyle $(1960,1972,1973)$ and by Crabbé (1967, as cited by Boyle, 1973, and by Thinès, Costall, \& Butterworth, 1991), who had worked directly under Michotte. Why some people do not experience phenomenal causality, or do not experience it all of the time, is unclear. The effect is real for most people, however, as has been shown by independent replications (Gordon, Day, \& Stecher, 1990; Millar, 1977; Schlottmann, 1987; Schlottmann \& Shanks, 1992; White, Corballis, \& Corballis, 1986, as cited in White, 1988; also see Johansson, von Hofsten, \& Jansson, 1980).

Michotte's theoretical claim was that this perception of causality was direct, unmediated by inference. He linked this claim with the further claim that it was not derived from experience, but innately determined. Michotte's view of the nature of perception is akin to that of Gibson (1967; see p. 142), who notes that he was "in strikingly near agreement" with Michotte (for discussion of these issues, see Costall, 1991).

The objections to Michotte's theoretical claim also rest on individual differences-especially on results suggesting that subjects' reports are influenced by prior knowledge (Gemelli \& Cappellini, 1958; Natsoulas, 1961). But experimental demonstrations of such experiential effects have been scarce (Gruber, Fink, \& Damm, 1957; Powesland, 1959) and can be explained as sensory adaptation, which would not trouble Michotte. Nevertheless, these findings are sometimes interpreted to mean that phenomenal causality is based on learning and inference (e.g., Bruce \& Green, 1990; Weir, 1978), a conclusion that goes further than the data.

Michotte himself did recognize that there could be experiential influences on phenomenal causality (e.g., p. 256). He did not consider, however, how experiential and perceptual factors would interact. Indeed, Michotte generally played down the role of experiential factors as much as possible.

\section{Limits of Previous Methodology}

Elimination strategy. Michotte conducted two basic types of investigations, both intended to eliminate or minimize the influence of prior experience. To demonstrate the generality of phenomenal causality, he presented single launch events to large numbers of naive observers. His more quantitative investigations involved only a few experienced observers, generally including members of his laboratory. Michotte presumed that naive observers lacked the opportunity to develop attitudes toward launch events, and that highly experienced observers would be capable of separating beliefs and perceptions.

The elimination strategy was not very successful, as is suggested by the individual differences in phenomenal causality noted above. Indeed, Michotte's procedures did not escape the acid criticism of Joynson (1971), who suggested that his results may have been heavily influenced by subjects' knowledge of the hypothesis and/or by the implicit suggestions in such questions as, "could you be a little more precise?"'

An alternative approach is adopted here. It may not be possible to exclude experiential effects, but they can be manipulated. This may provide some opportunity to disentangle the relative contributions of experiential and perceptual factors.

Choice methodology. The inconsistent results cited above may be amplified by ambiguities inherent in the use of categorical choice data. Nearly all investigators have adopted Michotte's procedures of categorical response. They have either obtained choices among prescribed response categories or have collected free verbal reports to be categorized by the experimenter.

Three problems, not mutually exclusive, arise with such categorical data. First, individual differences may exist among subjects evaluating the different cues; optimal conditions for one subject may be suboptimal for another, as already noted by Michotte (e.g., p. 111). Second, different subjects may employ different decision criteria for assigning cases to the causal or noncausal categories. These are likely to be affected by experimental procedure and instruction. Third, categorical data are not very sen- 
sitive. With categorical data, it is difficult to determine optimal conditions, especially across different apparatus. For all these reasons, Michotte's account of what constitutes an optimal launch event, on which subsequent workers have focused, may lack generality.

\section{Information Integration Theory}

The concepts of information integration theory (Anderson, 1974, 1981, 1982, 1991) introduce a new research focus to the study of phenomenal causality. This focus is on multiple determination, on how different cues are combined into a unified impression. Michotte had already stressed that phenomenal causality depended on the particular configuration of variables, but he typically studied their effects one at a time. The present approach overcomes this limitation.

The integration approach does not entail any commitment about the experiential as opposed to the perceptual basis of phenomenal causality. Both aspects must contribute to adults' reports of perceptual experience, and the integration framework allows the study of their joint operation in producing an impression of phenomenal causality.

Continuous response. In the present approach, we employed a continuous response measure. This was appropriate, since Michotte had already pointed out that observers experienced graded differences in goodness of the causal impression (e.g., p. 95). Continuous measures are more informative and powerful than the choice measures used in previous work on phenomenal causality. They also avoid the cited difficulties stemming from the operation of the decision criterion. Accordingly, subjects were asked to judge goodness of the causal impression on a continuous scale.

A continuous measure may seem odd, for, in naive causality, event $A$ is or is not the cause of event $B$. However, continuous judgments of causality make sense in two ways. First, people can be more or less confident that $A$ has caused $B$, even with all-or-none causation. Second, rarely is $A$ a necessary and sufficient cause of $B$-events normally have multiple causes, in which case $A$ can have more or less causal effect on $B$. Preliminary work (Schlottmann, 1987) showed that subjects considered both types of judgment meaningful.

Parametric variation. In the present study, we investigated how three variables conjointly influence the causal impression: temporal delay and spatial gap at the point of impact, and the ratio of the two objects' speeds. The stimulus values on these dimensions were chosen to cover the transition range between definite causality and definite noncausality. Only in this transition region will graded differences in goodness of the causal impression be found. This selection followed Michotte's own specifications fairly closely, supported by the preliminary work.

Each subject is exposed to multiple launch events covering the range of stimulus values. The pattern of judgments for this set of events provides an overall picture of how the goodness of the causal impression depends on the three stimulus dimensions. With this procedure, the judgment of any particular configuration can be seen in relation to the overall integration pattern and optimal launch events can be determined individually.

Model-based analysis. From the information integration perspective, the conjoint action of multiple cues is both the focus of empirical analysis and a base for theory. In previous studies, concern was with the consistency of some stimulus configuration to elicit a causal response across individuals. The present concern, in contrast, is with the processes by which each individual arrives at this response.

The integration framework distinguishes three broad stages of processing. In the valuation stage, the information value of each stimulus variable is assessed independently, and the physical cues are transformed into subjective, task-specific representations. In the integration stage, these separate representations are combined to form the causal perception. Finally, the perceptual experience is translated into a judgment response. The parameter values for the separate stimulus variables as well as the rule governing their integration can be determined from the overall pattern of judgments by virtue of functional measurement methodology (Anderson, 1974, 1981).

Individual differences. Individual differences may be located in the valuation or integration stage of the process. Different subjects may employ different rules to integrate the informational cues, a result that would raise problems for Michotte's position. Alternatively, the integration rule may be invariant, but the information value of a given cue may differ across subjects.

The working hypothesis, on the basis of an initial study (Schlottmann, 1987), is that the integration of gap and delay will obey an invariant averaging rule. Individual differences will have their locus in the valuation of the separate cues. This hypothesis is compatible with Michotte's ideas of an invariant perceptual structure.

To allow for individual differences, single subject design is employed with a large group of subjects. Thus, response patterns that vary across but are stable within individuals can be distinguished from mere response variability. In addition, the degree to which response patterns are consistent between individuals can be assessed.

Some individual differences may stem from different attitudes toward causality. With multiple stimulus presentations, as was well known to Michotte, the question of how the task is interpreted is very important. The predispositions brought to the experiment by naive observers cannot be known, but manipulating instructions may help control interpretation of the task. Accordingly, two instruction conditions were used in the present study, one for the customary judgments of causality, the other for judgments of naturalness.

\section{METHOD}

\section{General Design}

Subjects saw launching scenes like that of Figure 1 in which three factors were manipulated: the size of the spatial gap, the temporal 
delay at the point of impact, and the $A: B$ speed ratio of the objects. Each subject was exposed to a 4 (gap) $\times 4$ (delay) $\times 4$ (speed ratio) factorial design that yielded 64 different scenes.

Two instruction conditions were used. In one, the subjects judged their confidence that $A$ 's movement did or did not cause $B$ 's movement; in the other, the subjects judged the naturalness of the colli sion sequence. The confidence judgment was taken to represent Michotte's phenomenal causality. The naturalness judgment was expected to be influenced more by the physical reality of the visual events.

\section{Material}

Stimulus scenes were generated on a Macintosh SE computer with the VideoWorks II Animation software. Playback was controlled by the VideoWorks II HyperCard driver. During playback, the screen was updated every $17 \mathrm{msec}$. With this updating frequency, an appearance of smooth, continuous motion was achieved.

Each scene consisted of the following sequence of events: A gray $5 \times 5 \mathrm{~mm}$ square $(A)$ faded in on the left together with a black square $(B)$ of the same size in the middle of the screen, $6.75 \mathrm{~cm}$ to the right of $A$. The squares rested in their starting positions for $1.2 \mathrm{sec}$. Then the left square moved at the constant velocity of $49 \mathrm{~cm} / \mathrm{sec}$ toward the middle, stopping $2.1,1.4,0.7$, or $0 \mathrm{~mm}$ before square $B$. (This range was smaller than indicated by Michotte, because he had used objects much larger than usual for the studies manipulating spatial contiguity.) Square $B$ started to move after 170 , 119,68 , or $17 \mathrm{msec}$, with a constant velocity of $49,24.5,12.2$, or $6.1 \mathrm{~cm} / \mathrm{sec}$ for $6.75 \mathrm{~cm}$. These velocity combinations yielded $A: B$ speed ratios of $1: 1,2: 1,4: 1$, and $8: 1$. Both squares then stayed at rest for $1 \mathrm{sec}$ and faded out. A scene therefore lasted about 2.5 $3.6 \mathrm{sec}$.

Each subject judged six sequences of the complete set of 64 scenes. The orders for three sequences were randomly generated for each subject. The reverse sequences were used to counterbalance any effects of practice or order.

The experiment had two supplementary conditions. Before the main task, each subject was exposed to four replications of one of the three $4 \times 4$ subdesigns, with the level of the third factor fixed at a speed ratio of $4: 1$, a gap of $0 \mathrm{~mm}$, or a delay of $68 \mathrm{msec}$, respectively. These data were collected for the model estimation procedure, but were not used (see the Appendix). Type of subdesign was balanced across instruction conditions in the main task. This factor had no substantial effect on judgments and is not considered further.

Following the main task, each subject judged the subjective size of gap and delay for three replications each of the $4 \times 4$ (gap $x$ delay) subdesign with the speed ratio fixed at $4: 1$. These component ratings were collected to check for configural effects on cue values, as noted later in the discussion of the $17-\mathrm{msec}$ condition.

\section{Procedure}

The subjects were tested individually in one session of about $2 \mathrm{~h}$. They were free to take short breaks throughout. They sat about $0.9 \mathrm{~m}$ from the monitor $(12 \times 18 \mathrm{~cm})$ in the dimly lit experimental room. They were told to focus on the area around the impact point and to keep a constant distance from the screen. The subjects made their judgments by moving a mouse along an $11.1-\mathrm{cm}$ unmarked graphic rating scale displayed on the lower part of the screen. This scale had a resolution of 300 points. The instructions, display of stimulus scenes, and on-line collection of ratings were self-paced in a HyperCard environment.

The instructions for the causality and naturalness judgments were identically phrased, except when the tasks themselves were concerned. The subjects were first shown four intermediate examplesthat is, scenes with moderate gaps, delays, and velocity ratios. After each scene, they were asked, "Did it look like $B$ moved because $A$ hit it? Was $B$ 's movement produced by $A$ ? - Or did $B$ take off on its own?" or "Did the collision between $A$ and $B$ look natural to you? Could it happen this way?-Or did the collision look im- possible and artificial?"' The instructions stressed that the subjects were watching animation sequences in which "anything was possible" and that no "real" collision was ever involved. This instruction was developed to focus them on phenomenal causality rather than on a representation of concrete physical events.

The rating task was introduced thus: "Your task in this experiment will be to judge just how confident you are that $A$ caused or did not cause $B$ " or "just how natural a collision looks." The instructions included four anchor stimuli, to set up a frame of reference for the rating scale. The high anchors had no gap and no delay; the low anchors had the largest gap and largest delay. The subjects were told that, in the experimenter's judgment, these scenes were among the most and least causal (or natural) scenes that they would see, although they need not agree with her opinion at all. Then subjects practiced using the rating scale without feedback ( 10 trials in the main task, 16 in the preliminary subdesign). Practice and end anchors helped equate the response range across subjects, thereby facilitating the pooling of individuals with similar integration strategies. Anchor stimuli were repeated after the practice, and additional clarifications and practice were given on demand. Numerous experiments have shown this rating procedure with end anchors to be effective in eliminating nonlinear biases in the response scale (Anderson, 1982, chap. 1).

\section{Subjects}

Sixty UCSD undergraduates participated in the experiment to fulfill a course requirement. Gender was balanced across conditions. All subjects claimed to have normal or corrected-to-normal vision.

\section{RESULTS AND DISCUSSION}

The overall results can be summarized simply. The integration of the three cues followed an invariant averaging rule. The valuation of each cue, in contrast, showed large individual differences. These individual differences, however, exhibited a small number of distinct patterns.

A key to the analysis lay in the interaction between the two judgment conditions and individual differences. The two judgment conditions produced different response patterns, as was expected. Individual differences were expected as well, and each subject served in six replications to enable reliable individual analyses. Inspection of these individual analyses showed that subjects differed widely in the effect of speed ratio, which led to five distinctive response patterns. These grouped themselves meaningfully when considered together with the experimental effects of the two instruction conditions: Two of the individual difference groups mapped onto the modal response patterns elicited by the two instructions; the other three formed an orderly spectrum between these poles. It appeared that subjects had preexperimental predispositions for one or the other of the two kinds of judgments, and these predispositions interacted with the experimental instructions to produce the five response patterns.

\section{Initial Analysis: Integration Patterns for Instruction Conditions}

The mean results for the causality judgments are shown in the top row of Figure 2. Each panel shows the integration pattern for the listed pair of variables. The top left panel, for example, shows the integration pattern for gap and delay. Smaller gaps produced markedly better im- 


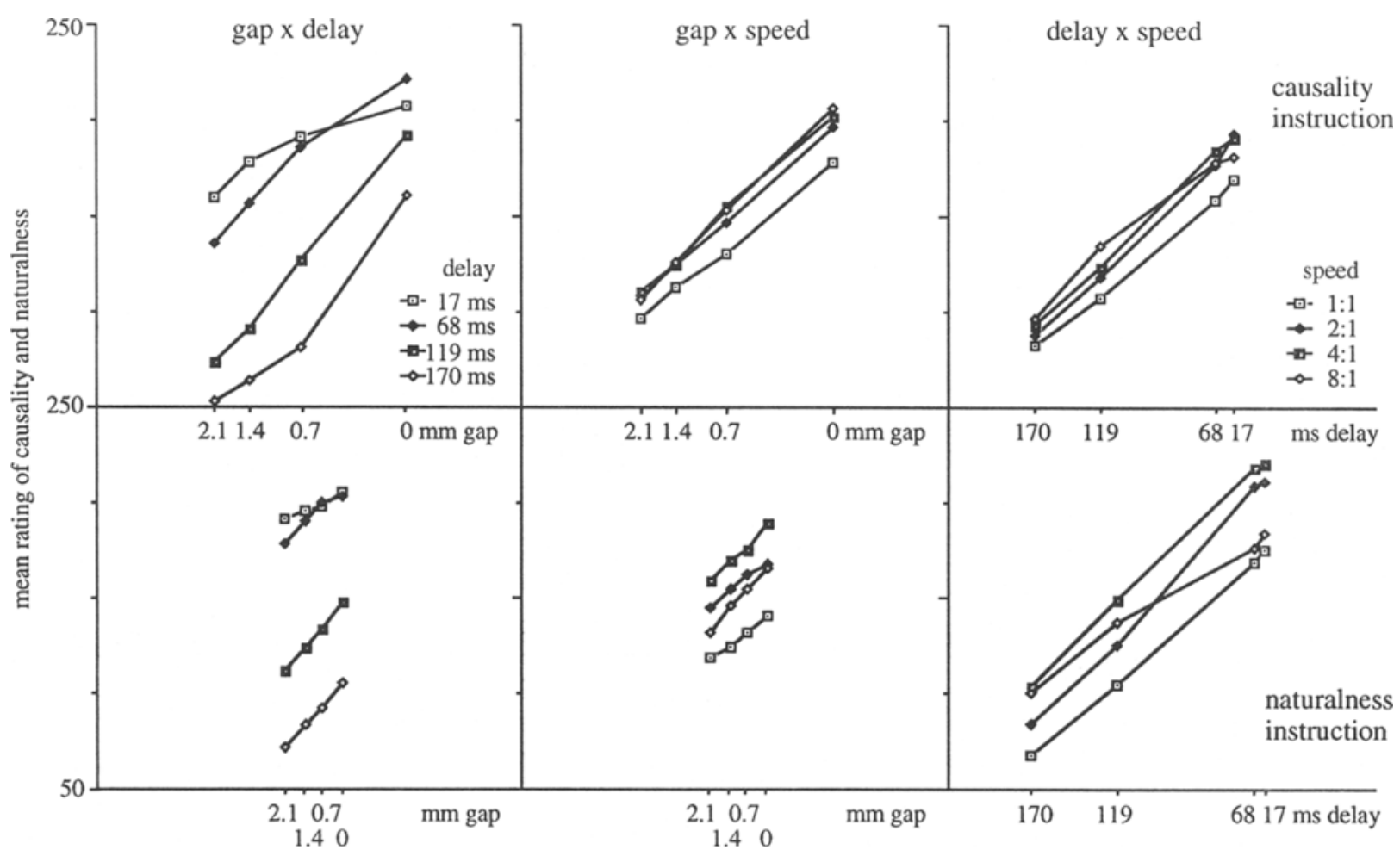

Figure 2. Mean judgments of causality/naturalness for two instruction conditions. The three panels in each row give the three twofactor views of the three-factor integration (see text).

pressions of causality, as is shown by the upward sweep of the curves. Shorter delays generally produced better causal impressions, as is shown by the vertical separation among the curves. Speed ratio (curve parameter in the middle and right panels) had only small effects, although larger speed ratios produced somewhat better causal impressions.

The naturalness judgments, in the bottom row of Figure 2 , show a different pattern. This group exhibits larger effects of speed ratio, as is shown by the larger separation among the curves in the bottom right panel, and large effects of delay, as is shown by the upward sweep of the curves. In contrast, gap had small effects, as is shown by the small horizontal extent of the curves in the bottom left and center panels (see the Appendix for statistical details).

An unusual mode of graphing was employed in Figure 2. Consider the two center panels. The four curves represent the four speed ratios for each instruction condition; the horizontal extent of the curves in each panel represents the effect of gap. This horizontal spacing is based on the marginal means of the gap factor in the design. In this way, the gap effect can be read off the horizontal axis, whereas slope is normalized. This mode of graphing was found to be more meaningful than the standard factorial graph in which multiple slopes were hard to assess.

\section{Integration Patterns for Individual Difference Groupings}

More finely grained analysis was achieved through inspection of the individual subjects' data. The individual subjects were found to differ widely in preferred speed ratio. The group differences seen in Figure 2 already pointed to speed ratio as a source of variation in judgment, but the group means oversimplified the results. Accordingly, the subjects were classed in five groupings on the basis of which speed ratio yielded the highest response. In Figure 3, each layer of the graph shows the results for one of these five groupings. The optimal speed ratio for each group is indicated by the arrows in the right column of Figure 3.

The patterns found in the top and bottom rows of panels in Figure 3 are strikingly similar to those found for the mean instruction conditions in Figure 2. Group 1 (top), with a large gap and negligible speed effect, exhibits the modal pattern of causality judgments. All but 1 of the 13 subjects in Group 1 were from the causality instruction condition. Group 5 (bottom) shows the modal pattern for naturalness judgments, with a small gap and large speed effect. All but 3 of the 14 subjects in this group received the naturalness instructions.

The remaining three groups show intermediate patterns. This grouping revealed the top-to-bottom trends visible in Figure 3; gap decreases in effect, whereas delay and speed 


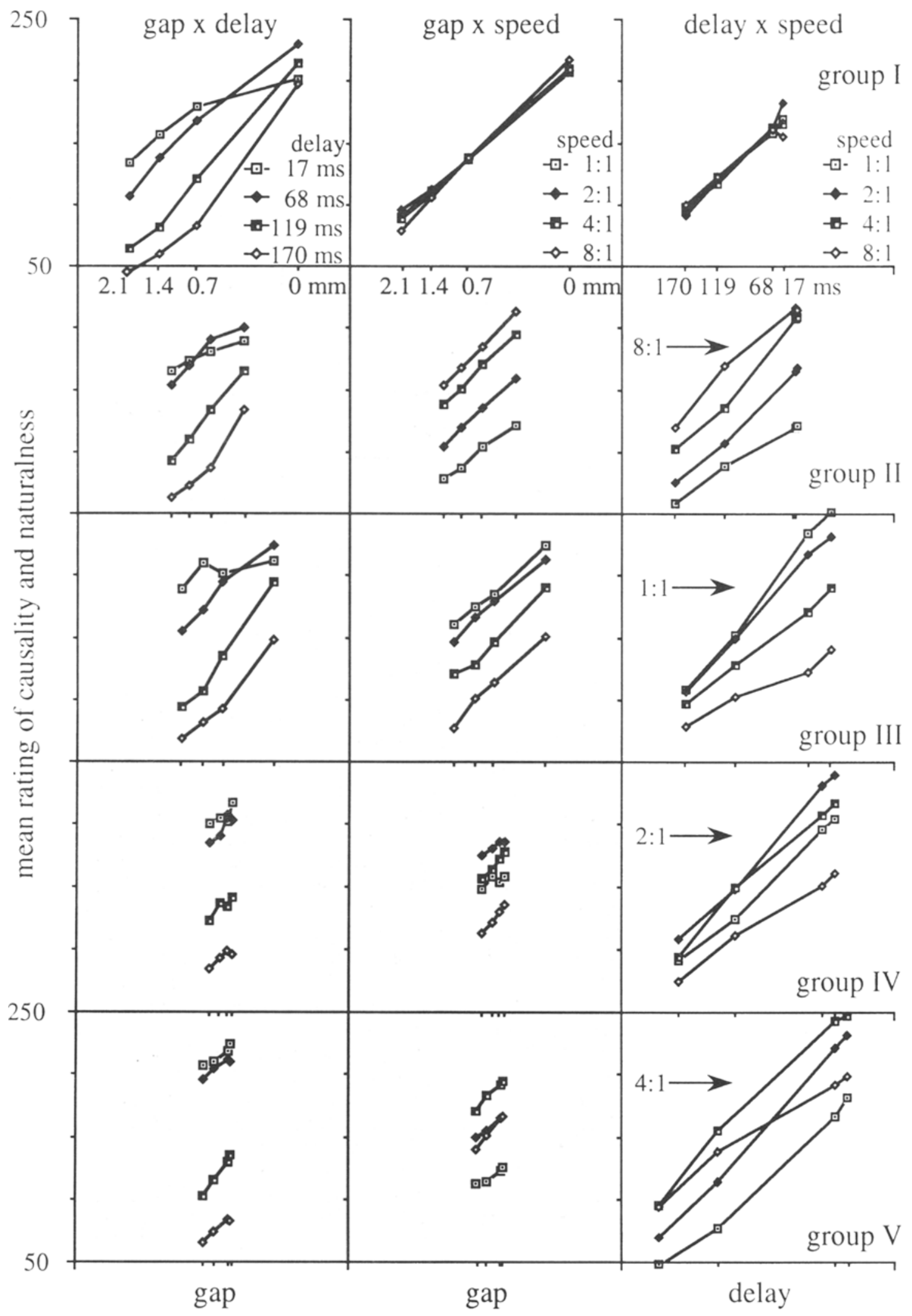

Figure 3. Mean judgments of causality/naturalness for five groups of subjects. Each row of the graph represents one group, classified according to which speed ratio yielded the highest judgments (see arrow in rightmost panels). 
Table 1

\begin{tabular}{|c|c|c|c|c|c|c|c|c|c|c|c|}
\hline \multirow[b]{3}{*}{ Condition } & \multicolumn{10}{|c|}{ Group } & \multirow[b]{3}{*}{ Total } \\
\hline & \multicolumn{2}{|c|}{1} & \multicolumn{2}{|c|}{2} & \multicolumn{2}{|c|}{3} & \multicolumn{2}{|c|}{4} & \multicolumn{2}{|c|}{5} & \\
\hline & No. & $\%$ & No. & $\%$ & No. & $\%$ & No. & $\%$ & No. & $\%$ & \\
\hline Causality & 12 & 92 & 10 & 56 & 3 & 38 & 2 & 29 & 3 & 21 & 30 \\
\hline Naturalness & 1 & 8 & 8 & 44 & 5 & 63 & 5 & 71 & 11 & 79 & 30 \\
\hline Total & 13 & 100 & 18 & 100 & 8 & 100 & 7 & 100 & 14 & 100 & 60 \\
\hline
\end{tabular}

ratio increase. The grouping is reliable, since it is based on 6 replications of the 64 scenes for each individual.

Speed ratio, it seems, is a rather different perceptual cue from the delay and gap variables. For delay and gap, values closer to zero are more causal, and subjects uniformly agreed on that. All speed ratios presented are possible, however, for this is determined by the relative masses of the objects and the elasticity of the collision (ignoring friction). The effect of speed ratio is only loosely constrained by physical considerations, and more dependent on individual predisposition and preference. The occurrence of individual subgroupings on this factor is thus not entirely surprising, although not foreseen on the basis of Michotte's reports.

\section{Causality, Naturalness, and Individual Differences}

The present analysis indicates that individual differences were related to the experimental instruction conditions. The two conditions elicit different information processing, as illustrated in Figure 2, and these differences reappear even more clearly in the individual difference grouping of Figure 3: Two groups reflect pure effects of the experimental manipulation, and the other three are intermediate in terms of the data patterns. They are also intermediate in terms of the distribution of subjects. The number of subjects judging causality decreases from Groups 1 through 5, while the number judging naturalness increases. These trends are shown in Table 1.

Instructions thus appear to have interacted with individual differences. The subjects seem to have had predispositions toward one or the other of the two instruction sets. A predisposition for causal judgments, for example, would be enhanced by causality instructions and depressed by naturalness instructions. Thus, causality instructions enhanced the role of spatial contiguity and depressed the role of the relative speeds; naturalness had the reverse'effect (see Figure 2). Nonetheless, some subjects in the causality condition, but predisposed toward naturalness, would have considered speed ratio. Conversely, some subjects in the naturalness condition, but predisposed toward causality, would have considered gap (see Figure 3). Hence, the effect of each stimulus cue depends partly on the instructions and partly on each subject's predisposition.

The meaningful coherence of individual differences and experimental instruction conditions supports the grouping in Figure 3. The present interpretation of individual differences in terms of predispositions, of course, cannot be checked in the present experiment; it would re- quire some initial measurement of predisposition. The main point here, however, is that the grouping adjoins individual differences to the experimental conditions. A more detailed analysis thus becomes possible, as will appear in the parameter estimates of Table 2 below.

\section{Integration and Valuation}

The hypothesis of an invariant integration rule is consistent with the taper patterns visible in Figure 3. In nearly every panel, the curves are wider in the middle, with a convergence toward one end or the other. In a few cases, the taper is bilateral, most notably in the upper left and lower right panels. This taper does not seem attributable to floor-ceiling effects, because the effective response range occurs only over the inner two thirds of the 300 point response scale. Instead, this taper suggests that the information is integrated by an averaging rule in every group (see the preliminary study below).

Under this hypothesis, the large differences in surface response pattern, so obvious in Figure 3, arise at the stage of cue valuation-that is, in the processing of each separate stimulus variable for its information content. Such differences in valuation are clear in the sharp differences in effect of gap and speed ratio across groups in Figure 3. Once evaluated, the cues are integrated by the same rule across all groups. Quantitative analysis to buttress this interpretation will be given in the section on model analysis below.

\section{Continuous Causality}

Continuous perception of causality was obtained by varying each stimulus dimension over the transition range between definite phenomenal causality and definite noncausality. Unambiguous scenes outside this transition range, with larger delays, for example, would have completely destroyed any impression of causality and wiped out all instruction and individual differences (Schlottmann \& Shanks, 1992).

The use of continuous judgments agrees with Michotte's observation of graded differences in goodness of causal impression. It is consistent with the use of psychophysical methods to determine a "threshold" for causal perception. A continuous response, actually a rating method, was also used by Gordon et al. (1990). The usefulness of this procedure, as already noted, rested in the selection of stimulus levels over the causal-noncausal transition ranges.

It is still desirable to check that individual subjects did indeed make continuous judgments. Group frequency dis- 
tributions are shown in Figure 4, which exhibits mild bipolarity for both instruction conditions. The group data, however, could mask bipolar, yes-no responding for individual subjects. Since each subject had judged each of the 64 scenes six times, a frequency distribution based on the 384 responses was available for each subject. Mild bipolarity was seen in about half the causality subjects and about a third of the naturalness subjects. All subjects, however, made responses more or less uniformly over the whole response range.

\section{Two Crossovers}

Two peculiarities of Figure 3 deserve mention. First, in the top left panel, the 17-msec delay curve is relatively flat, being the highest at the left, but almost the lowest at the right. This very short delay thus improves the causal impression for large gaps, but makes it worse for small gaps. Other panels in the first column show a similar, if smaller, effect. This agrees with Michotte's observation that a small temporal delay may improve phenomenal causality under certain conditions (p. 94), a finding that emphasizes that phenomenal causality is not physical causality.

This flattening of the 17-msec curve is reliable (also see Figure 5 for the preliminary study below). It appears as a significant gap $\times$ delay interaction in a statistical comparison of the 17- and 68-msec delay curves across all five groups $[F(3,177)=15.13]$. Visual inspection shows that this interaction takes crossover form mainly in Groups 1-3.

This flattening and crossover of the $17-\mathrm{msec}$ curve may result from a physical stimulus interaction. The gap is

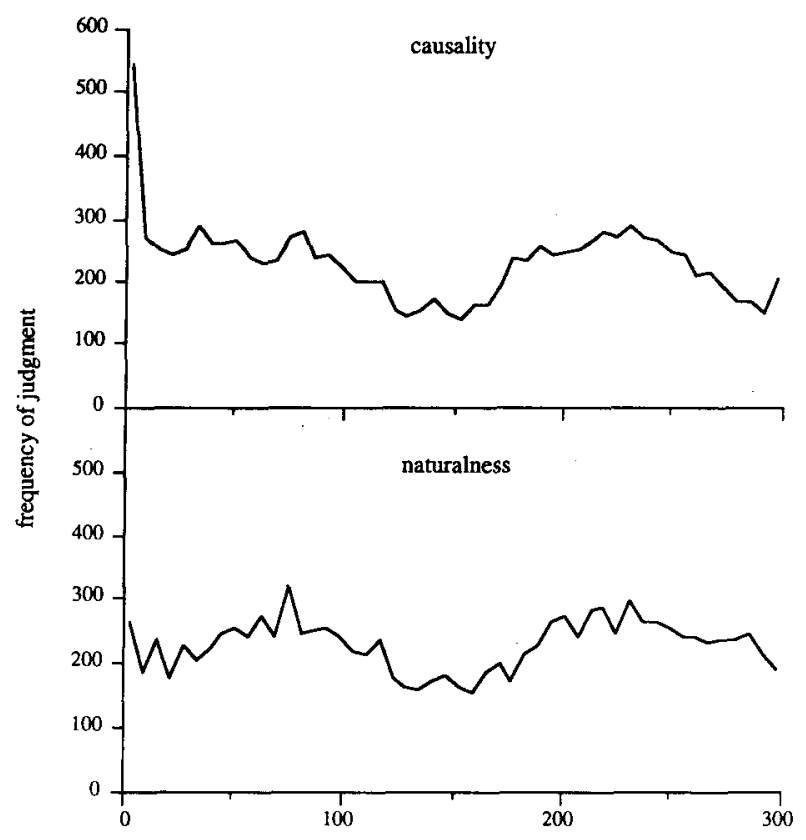

Figure 4. Group frequency distributions for 30 subjects judging causality and 30 subjects judging naturalness. Each graph contains 11,520 total observations. properly visible only during the delay, and the length of the delay accordingly limits the information physically available about the gap. Full apprehension of the gap may not be possible within $17 \mathrm{msec}$, and this would lead to a reduced influence of gap on the overall judgment. In fact, such reduced effect would correspond to the relatively shallow slope of the 17-msec curves in the upper left panels of Figure 3.

This interpretation in terms of a physical interaction is supported by the supplementary data on direct judgments of the size of the gap. These gap judgments ranged from 110 to 195 in the $17-\mathrm{msec}$ delay condition. The corresponding ranges in the longer $68-, 119-$, and $170-\mathrm{msec}$ conditions were 86-256, 54-268, and 45-274, respectively. Discrimination was mainly reduced at the shortest delay, with a gap effect half or less in size than that found at the longer delays. The crossover of the $17-\mathrm{msec}$ curve in the left panels of Figure 3 thus appears to stem from a physical interaction of gap and delay information available in the stimulus event.

The second crossover appears in the lower right panel, in which the 8:1 speed ratio curve is highest at the left, but next to lowest at the right. A high speed ratio thus improves the causal impression for long delays, but depresses it for short delays. A post hoc test between the $8: 1$ and $4: 1$ curves for this group yielded a highly significant speed ratio $\times$ delay interaction $[F(3,39)=24.00]$. Visual inspection shows similar flattening of the 8:1 curve for Groups 3 and 4.

This statistical interaction corresponds to a reduced effect of delay at the 8:1 speed ratio, but there is no reason to suspect physical interaction. First, information about the length of the delay is equally available at all speed ratios. Second, not all groups show this interaction. The crossover of the 8:1 curve in Group 5 is thus considered to arise at a later stage of processing. This interpretation agrees with the averaging rule of cue integration.

\section{Preliminary Study}

The present study replicates and extends a preliminary experiment (Schlottmann, 1987). Both obtained similar patterns, as can be seen by comparing Figure 3 with Figure 5 , which presents the gap-delay integration data of the preliminary study. Of special importance is the taper pattern, found in all six panels. This pattern led to the present hypothesis of an invariant integration process.

The six panels of Figure 5 are for three instruction conditions in which subjects estimated (1) confidence that $A$ caused $B$; (2) the magnitude of $A$ 's effect on $B$; and (3) the realism or naturalness of the movement sequence. Each subject judged two sets of scenes in balanced order: Set $A$, in which $A$ went faster than $B$, and Set $B$, in which $A$ went slower than $B$.

The manipulation of instructions produced effects comparable to those found in the present study. The subjects' judgments of confidence and of the magnitude of $A$ 's effect (top and middle panels of Figure 5, respectively) were quite similar to each other. In the present study, accordingly, only the confidence instruction was retained. Real- 


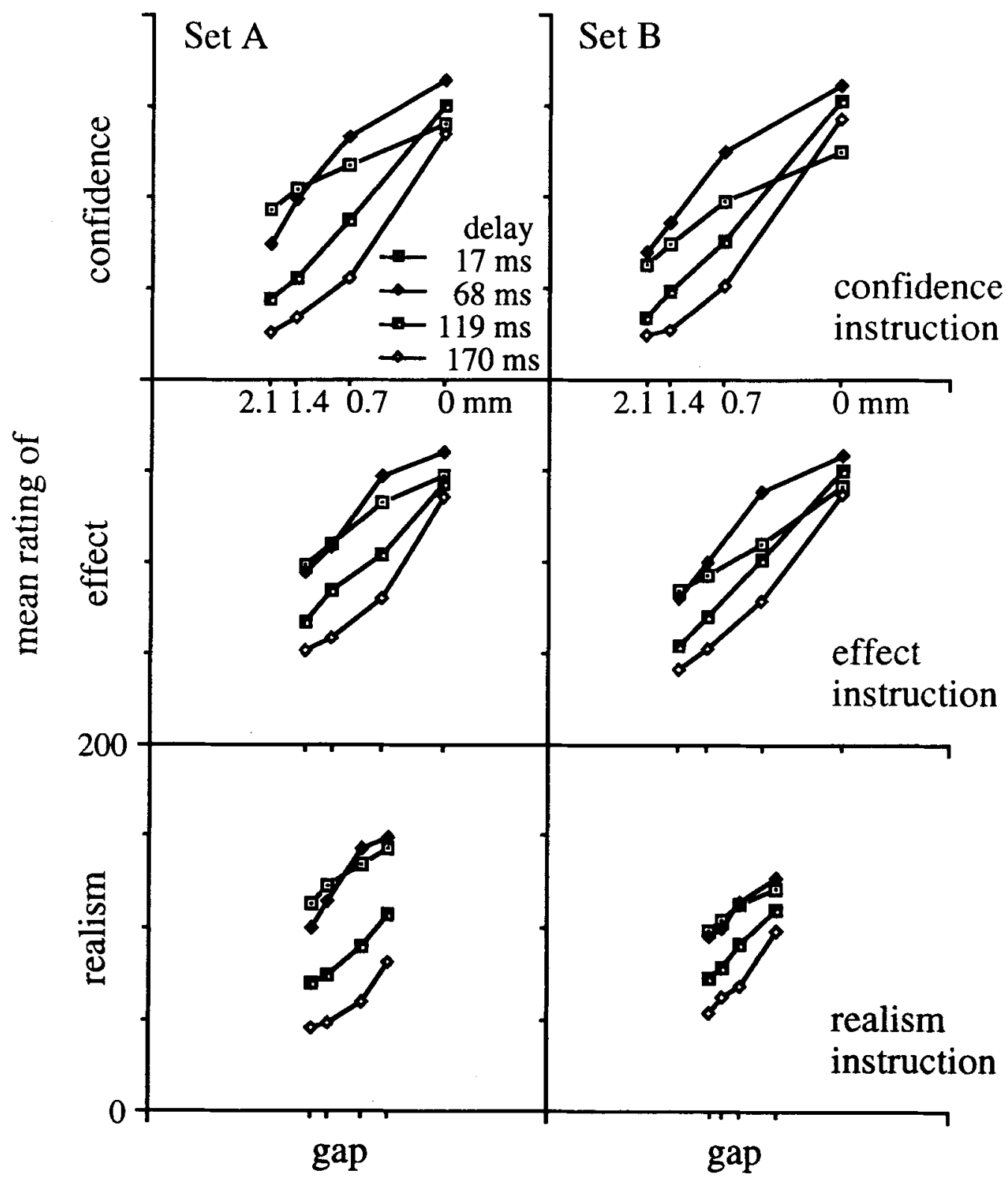

Figure 5. Gap-delay integration patterns of the preliminary study. The three rows of the graph represent subjects in three instruction conditions; the two panels in each row correspond to two stimulus sets.

ism judgments (bottom panels), in contrast, showed a relatively small effect of gap, a result replicated in the present study.

Speed ratio had small effects, but this seemed to reflect a less appropriate manipulation. In Set $A$, object $B$ always moved at the same low speed, and $A$ 's speed was scaled up to vary the ratio. Mean speed thus was less than half that in the present study, thereby reducing the effect of the speed ratio. In addition, the first object had variable speed across trials, which made comparisons of speed ratios difficult. In the present study, accordingly, $A$ always moved at the same high speed while $B$ 's speed was scaled down to manipulate the speed ratio, and this produced larger effects of speed ratio. Both studies agree, however, that the effect of speed ratio is easily affected by contextual factors.

\section{MODEL ANALYSIS}

In this section, the data are analyzed in terms of the averaging model of information integration theory. To illuminate the issue, it may help to show first how two additive models are not helpful to an understanding of the data. 


\section{Integration Models}

Additive model. The simplest model would specify the judgment, $r$, as an additive integration of the three informational cues:

$$
r=\psi_{g}+\psi_{d}+\psi_{s}
$$

where $\psi_{g}, \psi_{d}$, and $\psi_{s}$ are the information values for gap, delay, and speed ratio, respectively. Despite its simplicity, this model is fairly powerful because the $\psi$ values are treated as subjective parameters, with no fixed relation to the physical cues. This is important, because these $\psi$ values may thus embody individual differences in the processing of the physical cues. Hence the model may be applied separately to the five groups already discussed in Figure 3. It captures the main effects in the data.

The additive model, however, makes strong predictions at variance with the data. First, it predicts no crossovers, contrary to the lower right panel of Figure 3 . Second, on the assumption that the response scale is linear, which seems reasonable in light of previous work on functional measurement, the additive model predicts parallelism, whereas most panels in Figure 3 show marked taper patterns.

To account for the nonparallel data, the additive model could incorporate a configural parameter, denoted by $c_{g d s}$, which is added to the main effects of the three cues:

$$
r=\psi_{g}+\psi_{d}+\psi_{s}+c_{g d s}
$$

Here the effects of the informational cues differ according to the particular informational configurations in which the cues are presented. For example, a long delay might appear even less causal in the context of an unfavorable speed ratio than in the context of a favorable speed ratio.

This configural model, unfortunately, is not testable, because it can always fit the data perfectly. Mathematically, there is a free parameter, $c_{g d s}$, for each combination of cues. There are other ways to incorporate configural processing into strict adding models, of course, especially if one considers pairwise configurality among the three cues. Psychologically, however, there is no obvious way to specify the form of the configural processing so as to restrict the number of free parameters.

Averaging model. A more useful account of the data can be obtained with the averaging model of information integration theory, which has been used successfully in a number of areas of psychology (Anderson, 1981, 1982, 1991). The averaging model incorporates configurality, together with an adding-type integration. Formally, the overall judgment is a weighted average of the three informational cues:

$$
r=\frac{\left(w_{g} \psi_{g}+w_{d} \psi_{d}+w_{s} \psi_{s}+w_{0} \psi_{0}\right)}{\left(w_{g}+w_{d}+w_{s}+w_{0}\right)}
$$

Each stimulus level has its own scale value, $\psi$, and its own weight, $w$. Thus, $\psi_{g}$ denotes the value of each level of the gap variable on the response dimension, and so forth. The symbols $w_{0}$ and $\psi_{0}$ represent an initial expectancy that is standard in averaging theory, although not a present concern.
A separate weight parameter is used for each cue level, because cues may differ not only in value, but also in informativeness or diagnosticity, and this is represented in the weight. A small gap, for example, may be more diagnostic than an intermediate gap, because it points more definitely toward causality. A large gap, on the other hand, may also be more diagnostic than an intermediate gap, because it points definitely toward noncausality. If different cue levels carry different amounts of information, weights may not be equal or stand in a simple monotonic relation to value. In general, extreme cue levels are often more informative than intermediate levels. Hence, extremity weighting would be expected, with higher weights for both high and low than for intermediate cue levels.

This two-parameter, $w-\psi$, representation of the physical cues corresponds to two psychologically distinct aspects of processing. The value parameter reflects aspects of the psychophysical stimulus dimension; the weight parameter, $w$, reflects attentional or decisional aspects. This two-parameter representation makes the averaging model qualitatively different from an additive model, in which weight parameters are not generally identifiable.

Configurality in the averaging model. Configurality arises in the averaging model when different levels of a stimulus variable have different weights. In this case, the sum of the weights in the denominator of Equation 3 becomes variable for different configurations of cues. Because of this configural property, the averaging model can account for certain patterns of nonparallelism.

This configurality is conceptually and mathematically constrained, however. Mathematically, each level of a given cue must have the same fixed weight across all configurations. The weight for each level of delay, for example, is the same across all $4 \times 4$ cells of the gap $\times$ speed ratio design. This limits the number of free parameters and makes the model testable. Unlike the configural additive model, therefore, the averaging model cannot account for all patterns of nonparallelism.

Conceptually, the configural process is localized in the integration stage of processing. This constrains the applicability of the model. The averaging model may be used to account for nonparallelism produced by psychological interaction. Physical interaction, however, requires a model that allows for configurality at the prior valuation stage of processing. This is not allowed in the present version of the model.

Averaging with extremity weighting predicts taper patterns as seen throughout Figure 3 . To illustrate, consider a medium-weight cue combined either with a nondiagnostic, low-weight cue or with a diagnostic, high-weight cue. The denominator of Equation 3 is smaller in the former than in the latter case. Hence, the medium-weight cue contributes more to the overall judgment in the former than in the latter case. In this way, the averaging process automatically deemphasizes nondiagnostic cues and emphasizes diagnostic cues.

While the taper suggests invariant averaging integration, the individual difference in the curve patterns of Fig- 
ure 3 are taken to arise during valuation processing. Subjects may differ, in other words, in the weight-value representation for the stimulus cues. The particular parameter structure for each of the five groups can be clarified through model estimation, described in the following section.

\section{Averaging Theory Analysis}

Model fitting. The parameters of the averaging model were estimated with the use of the AVERAGE program (Zalinski \& Anderson, 1986, 1991). Since the averaging model does not apply to physical interaction, as noted above, the data for the $17-\mathrm{msec}$ delay were omitted in the model analysis. This left 48 data points for the estimation. With the present design, weight parameters for each informational dimension are mathematically identifiable up to a linear transformation (Anderson, 1982, Section 2.3.2).

The model was fitted separately for each of the five groups. Since the speed effect was very small in Group 1, an additional constraint was imposed; namely, weights for all levels of speed ratio were fixed at 1. For Groups 4 and 5, similarly, weights for gap were fixed at 1 (see the Appendix).

Theoretical predictions from the averaging model are given in Figure 6, which has the same format as does Figure 3 . The curves show the predictions, the points show the observed data. The overall fit is good: The mean magnitude deviations between predicted and observed are 5.0, $4.7,5.0,6.5$, and 4.4 on the 300 -point scale range in Groups $1-5$, respectively. The qualitative trends are captured well. In particular, each set of predicted curves shows the same taper pattern as do the data. Notably, the model also accounts for the crossover in the lower right panel.

Value estimates. Value estimates are found in the columns headed $\psi$ in Table 2 . A $\psi$ value around the midpoint of 150 may be regarded as neutral; larger values represent favorable or good values on the dimension of judgment; smaller values represent unfavorable or bad values.
Value estimates for delay are shown in the middle layer of Table 2. These show a uniform trend across all groups: high values for short delays and low values for long delays, in line with phenomenology. Value estimates for gap are shown in the bottom layer of Table 2 . These also show a uniform trend across groups: high values for small gaps and low values for large gaps, also in line with phenomenology. This trend (with one minor inversion) appears even in Groups 4 and 5, where the overall effect of gap was small. These values thus provide a quantitative representation of the psychophysical effects of the delay and gap variables.

Value estimates for speed ratio are shown in the top layer of Table 2. In Group 1, these estimates are all near 150 , the neutral point of the response scale, which reflects the virtually complete lack of speed effect in this group. For each remaining group, the highest value corresponds to the highest speed ratio curve, indicated by the arrow in Figure 3, whereas the lowest value corresponds to the lowest curve. For this perceptual dimension, therefore, the subjective values show different ordinal dependence on the physical speed ratio in different groups. Groups 2 and 3 actually show opposite ordering.

Weight estimates. Estimates of the weights, which measure diagnostic power, have primary theoretical interest. These estimates point to a general "good-bad" strategy: Subjects used one informational dimension to define "good" launch events, another to define "bad" launch events.

This good-bad strategy can be illustrated with the weight estimates for Group 1, leftmost in Table 2. Weights for the gap dimension are found in the bottom layer. These range from a high of 9.2 for the largest gap to a low of 2.6 for no gap. Since large gaps have low scale values, the high weight means that the gap is primarily used to diagnose bad causal events. But there is no corresponding tendency to use small gaps to diagnose good causal events, because the weights show no corresponding increase for small gaps.

Group 1 shows an opposite good-bad strategy for the delay dimension in the middle layer. The weight of 3.8

Table 2

Weight and Value Estimates for Three Cues from Averaging Model

\begin{tabular}{|c|c|c|c|c|c|c|c|c|c|c|c|c|}
\hline \multicolumn{3}{|c|}{$\begin{array}{r}\text { Cue } \\
\end{array}$} & \multicolumn{10}{|c|}{ Group } \\
\hline \multirow{2}{*}{$\begin{array}{l}\text { Speed } \\
\text { Ratio }\end{array}$} & \multirow[b]{2}{*}{ Delay } & \multirow[b]{2}{*}{ Gap } & \multicolumn{2}{|c|}{1} & \multicolumn{2}{|c|}{2} & \multicolumn{2}{|c|}{3} & \multicolumn{2}{|c|}{4} & \multicolumn{2}{|c|}{5} \\
\hline & & & $w$ & $\psi$ & $w$ & $\psi$ & $w$ & $\psi$ & $w$ & $\psi$ & $w$ & $\psi$ \\
\hline $1: 1$ & & & 1 & 151 & 7.9 & 87 & 3.5 & 186 & 5.6 & 158 & 6.9 & 126 \\
\hline $2: 1$ & & & 1 & 145 & 4.7 & 122 & 3.9 & 177 & 5.5 & 180 & 5.5 & 165 \\
\hline $4: 1$ & & & 1 & 154 & 3.7 & 166 & 5.0 & 146 & 5.8 & 170 & 5.6 & 188 \\
\hline $8: 1$ & & & 1 & 167 & 3.9 & 189 & 7.2 & 108 & 5.9 & 136 & 8.4 & 160 \\
\hline & 68 & & 7.4 & 167 & 2.9 & 240 & 1.6 & 206 & 2.9 & 180 & 4.1 & 235 \\
\hline & 119 & & 4.6 & 124 & 1.7 & 107 & 2.5 & 97 & 5.5 & 120 & 2.4 & 99 \\
\hline & 170 & & 3.8 & 100 & 2.2 & 19 & 5.7 & 66 & 7.1 & 79 & 4.5 & 48 \\
\hline & & 0 & 2.6 & 240 & 4.5 & 199 & 5.7 & 236 & 1 & 187 & 1 & 202 \\
\hline & & 0.7 & 4.5 & 151 & 2.7 & 158 & 2.4 & 178 & 1 & 192 & 1 & 192 \\
\hline & & 1.4 & 6.4 & 113 & 3.0 & 122 & 1.8 & 133 & 1 & 152 & 1 & 135 \\
\hline & & 2.1 & 9.2 & 86 & 3.2 & 97 & 1.6 & 96 & 1 & 112 & 1 & 86 \\
\hline
\end{tabular}

Note-Delay is given in milliseconds; gap, in millimeters. 


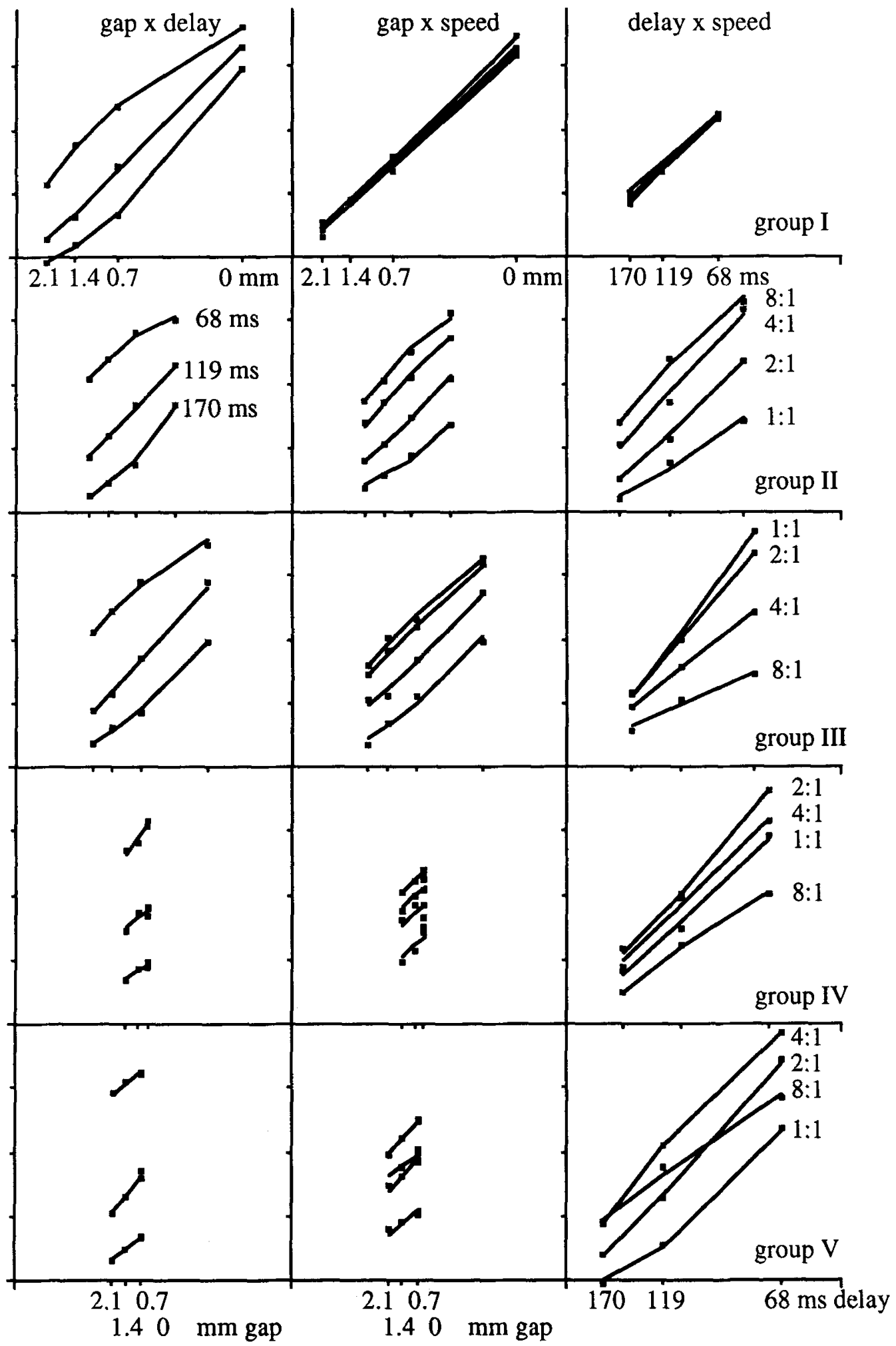

Figure 6. Comparison between predictions of the averaging model (curves) and the observed data (points). This graph has a format and data points identical to those in Figure 3, except for the omission of data for the 17-msec delay. 
for large delays is smallest, and weights increase with shorter delays. Group 1 thus uses the delay dimension to define goodness of an event.

Here, as elsewhere, it should be recognized that the design only allows a linear scale for weights (see the Appendix). Weights may thus not be comparable across dimensions, but differences between weights within each dimension are meaningful. The logic of the present "goodbad" interpretation rests on the weight trends within each cue dimension for each group.

A different strategy can be seen in the rightmost column for Group 5, which judged naturalness and ignored gap. Weight estimates for delay are found in the middle layer of the right column. Here, both extremes have received higher weights, 4.1 and 4.5 , whereas intermediate delay has a smaller weight of 2.4. Group 5 thus used the delay dimension to select both good and bad events.

Weight estimates for speed ratio for Group 5 are in the top layer. From Figure 2, Group 5 considered a speed ratio of 4:1 most natural, 1:1 least natural. Since 1:1 received a higher weight than $4: 1$, speed ratio was used to define bad events. Note that $8: 1$ received the highest weight on the speed dimension, which reflects the flatness and crossover of the 8:1 curve in Figure 2. This is not an extremity effect, however, since 8:1 does not have a high scale value as well.

Indications of similar good-bad strategies can be seen in the weight estimates for the other groups. A glance over all groups, however, will show that the selection of good and bad dimensions differs across groups.

One important implication of these different good-bad strategies is that the valuation processes that extract the cue information have a cognitive component. Purely perceptual considerations would imply a pattern of weighting tied to the pattern of $\psi$ values-that is, higher weights for both higher and lower stimulus levels, and lower weights for the less diagnostic intermediate levels. In contrast, in the present good-bad strategies, the diagnostic function of the stimulus variables does not reflect their psychophysical content.

These parameter estimates reveal aspects of processing that could not be understood from the data patterns themselves. The model application is tentative, but the results suggest the potential of the approach. The taper patterns in the judgment data suggested differential weighting, but did not localize it; that required the model analysis.

\section{CONCLUSIONS}

The averaging model of information integration theory gives a good account of Michotte's phenomenal causality. The model provides a clear distinction between two processing stages, valuation and integration. Although individual differences were large, they could be localized in the valuation operation that constructs the parameters of the integration model. Thus, different subjects extracted quite different informational content from the same stimulus variable, but all integrated the information by a uniform averaging rule.
The present account allows some reconciliation between Michotte and his critics: The averaging integration model may correspond to the invariant perceptual structure of phenomenal causality, as proposed by Michotte. The valuation operation, on the other hand, can accommodate individual differences that may have experiential components, as suggested by his critics.

\section{Good-Bad Strategy}

Characteristic of the valuation operation was a "goodbad" strategy in cue weighting. For the most part, subjects treated each stimulus dimension either as "good," giving higher weights to stimulus levels considered more indicative of a causal relation, or as "bad," giving higher weights to stimulus levels more indicative of a noncausal relation. Detection of this good-bad strategy depended on the model parameter analysis, which can separate the weight parameter from the scale value, a singular capability of the averaging model.

The good-bad strategy points to a cognitive level of processing in phenomenal causality. Psychophysical considerations, in line with phenomenology, can account for the pattern of $\psi$ values. But the weighting pattern of the goodbad strategy was not tied to this pattern of the values. Thus, the weighting structure involves additional operations, presumably reflecting the experience of the observers.

\section{Individual Differences and Instruction Conditions}

Individual differences played a unique role in the analysis, a role actualized by the two instruction conditions. As expected, instructions to judge naturalness yielded a different response pattern from instructions to judge causality. Study of the individual patterns revealed two corresponding modal patterns, one for causality and one for naturalness. Between these two modal patterns were found three intermediate patterns, each characterized by a different optimal level of the speed ratio variable.

Instead of the customary analysis at the level of the instruction conditions, therefore, a more meaningful analysis could be obtained at a finer level of individual difference groups. This revealed aspects of the response patterns that were obscured in the overall analysis of the two instruction groups. It was this breakdown, coupled with the model analysis, that localized the individual differences in the valuation operation, followed by the same integration operation across subjects.

The value of the two sets of instructions deserves emphasis. Subjects appear to have predispositions to one of the two response sets, causality or naturalness. The present instructions were carefully developed in the preliminary work, yet individual differences were found within these instruction conditions. Some of the inconsistencies in previous work may reflect differences in instructions, which have not previously received much attention. With experimental manipulation of instructions, these individual differences can be broken down meaningfully.

This conjoint use of instructions and individual differences may have more general applicability as an analytical tool. Individual differences have been noted by many 
experimental psychologists, and Underwood (1975) has suggested that they should be a crucible for theory construction. Underwood's argument is attractive, but it has remained largely an aspiration. Too often, the criticism that experimental psychology consigns individual differences to the error variability is justified. In the present study, individual differences are adjoined to experimental conditions. This technique illustrates a potentially general method for utilizing individual differences productively.

\section{Phenomenal Causality and Individual Differences}

The present results may provide at least some agreement between Michotte and his critics. The model analysis indicates a uniform integration process, the same across different individuals. This integration process leads directly to the phenomenal experience, which is the integrated resultant. Although Michotte did not pursue the issue of cue integration, he emphasized that the phenomenal experience would depend on the combined action of operative cues. The averaging model gives precision to this integration problem. It is not an additive model, but it captures one aspect of cue configurality through the relative weights. Tentatively, therefore, it seems reasonable to relate Michotte's concept of a uniform perceptual structure directly to the integration rule.

The large individual differences agree with Michotte's critics. Indeed, they provide more definite evidence than has previously been available, and they could be specifically related to cue valuation processes. But the implications of these individual differences for Michotte's claims need to be reconsidered.

Individual differences in $\psi$ values could be attributed in part to predispositions to judge causality or naturalness. Perhaps this is not too surprising. Both ratings are meaningful, and if philosophers cannot agree on how to view causality, one should not expect this from casual observers. Michotte had already observed an "analytic attitude" in some subjects, but dismissed such subjects as deviant. In theory, he recognized that "attitudes" could affect phenomenal causality (e.g., p. 333), but this was not relevant to his theoretical concerns; in practice he considered such effects negligible. This assumption can be dropped in the present approach, which allows a range of attitudes toward causality, as shown in the five-group breakdown of Figure 3.

The individual differences in cue weighting suggest a greater problem for Michotte's view. The real problem is not that there are individual differences, but their pattern: They follow the "good-bad" strategies described above. Even the delay variable, which showed relatively small individual differences in $\psi$ values, exhibited such weighting strategies. These seem to involve a cognitive level of processing, in disagreement with Michotte.

A possible resolution is to separate Michotte's twin claims of direct perception and of innateness. Individual differences, as in the "good-bad" strategies, hardly seem consistent with innate perception, but they need not raise a problem for direct perception. A similar separation has been suggested by Costall (1991, p. 58) in an overview of Michotte's work. In this approach, the present results contribute to the explication of how experiential factors influence perception, a point acknowledged but not pursued by Michotte.

\section{Phenomenal Causality and Effects of Experience}

One major alternative approach to phenomenal causality goes back at least to Hume (1739/1978), who proposed that causal beliefs are acquired through repeated experience of the constant conjunction of essentially independent events. Many contemporary studies have indeed shown that causality judgment is sensitive to the predictive relationships between events (for an overview, see Shanks, 1993; Wasserman, 1990).

This work, however, has little bearing on the perceptual task chosen by Michotte to exhibit direct perception of causality. In the one attempt to manipulate predictive relations within sequences of launch events, learning effects on phenomenal causality were not found (Schlottmann \& Shanks, 1992).

A different approach to experiential modification of phenomenal causality in the launch event is suggested by the good-bad strategies indicated by the present model analysis. These weighting strategies seem to have cognitive components and thus should be modifiable through experience.

One direct form of experiential modification could consist of preliminary training to influence these strategies. This might be done by holding a selected cue constant in an initial phase, while the other two were varied jointly. The present Group 1, for example, used the gap cue to define bad causal events. With the gap held constant, however, it could not be used as a basis for the bad strategy. Instead, strategies would have to have been based exclusively on the other two cues. Such effects might be maintained when the gap variable was faded in during a subsequent test phase in which all three cues were varied.

A related test is suggested by Groups 4 and 5, who considered the gap essentially irrelevant. By holding delay constant in an initial phase, these subjects might be impelled to take the gap into account, for otherwise they would have to rely entirely on the speed ratio. Alternatively, an initial three-cue phase like that in the present study might be used to diagnose good-bad strategies, followed by directed two-cue training, with subsequent transfer back to the three-cue task. The averaging model may be used to analyze the structure of any such learning effects on phenomenal causality in terms of weight and value parameters.

\section{The Continued Relevance of Michotte's Ideas}

Michotte pointed out a basic aspect of everyday phenomenology - that certain instances of causality are intuitively obvious. The very artificiality of the stimulus displays for launch events strengthens this point. Michotte was the first to tackle the problem of phenomenal causality in 
a systematic way, and his work is notable for the attempt to combine phenomenology and empirical quantification.

Subsequent workers have fixated on Michotte's claim that phenomenal causality is innate. Some have emphasized the phenomenal immediacy of the effect and its independence of physical reality; others have stressed the effects of prior experience and individual differences. The results have been inconclusive. Little more has been added to Michotte's work than that individual differences exist. Michotte had already acknowledged this, but his invocation of an "analytical attitude" was not a satisfactory conceptualization of the processes involved. The present approach allows for more detailed study of phenomenal causality at the individual level.

The integration approach returns to Michotte's focus on the phenomenon, especially to his concern with parametric variation along relevant stimulus dimensions. Through the incorporation of a continuous response measure, the usefulness of Michotte's task could be increased. It deserves reemphasis that the parameter specifications reported in Michotte's extensive studies were generally well supported in the present work.

The innate-versus-learned controversy is finessed in the present approach. This dichotomy has become increasingly simplistic in light of recent developmental work, which shows innate organization for causality in infants (e.g., Leslie, 1988). This does not preclude experiential learning. Indeed, some consider such scaffolding a learning device (e.g., Gelman, 1990; Leslie, 1988). In adult cognition, at any rate, the perceptual illusion of phenomenal causality must function together with acquired knowledge about causality in the physical world. Thus, ways are needed that can make effective progress on the innateplus-learned question.

Information integration theory is one useful tool in this endeavor. The complete pattern of individuals' impressions can be obtained via continuous responses to parametric variation of multiple cues over the causality-noncausality transition range. Analysis of these patterns provided evidence for an invariant integration model underlying phenomenal causality. At the same time, this model-based approach enabled a more principled consideration of individual differences. Thus, the integration framework provides the potential to study phenomenal causality within everyday cognition.

\section{REFERENCES}

Anderson, N. H. (1974). Algebraic models in perception. In E. C. Carterette \& M. Friedman (Eds.), Handbook of perception (Vol. 2, pp. 215-298). New York: Academic Press.

ANDERSON, N. H. (1981). Foundations of information integration theory. New York: Academic Press.

ANDERSON, N. H. (1982). Methods of information integration theory. New York: Academic Press.

ANDERSON, N. H. (ED.) (1991). Contributions to information integration theory ( 3 vols.). Hillsdale, NJ: Erlbaum.

BEASLEy, N. A. (1968). The extent of individual differences in the perception of causality. Canadian Journal of Psychology, 22, 399-407.
BoYLE, D. G. (1960). A contribution to the study of phenomenal causation. Quarterly Journal of Experimental Psychology, 12, 171-179.

Boy Le, D. G. (1972). Michotte's ideas. Bulletin of the British Psychological Society, 25, 89-91.

BoyLe, D. G. (1973). Letter. Bulletin of the British Psychological Society, 26, 82.

Bruce, V., \& Green, P. R. (1990). Visual perception. (2nd ed.). Hillsdale, NJ: Erlbaum.

Costall, A. (1991). Phenomenal causality. In G. Thinès, A. Costall, \& G. Butterworth (Eds. \& Trans.), Michotte's phenomenology of perception (pp. 51-64). Hillsdale, NJ: Erlbaum.

Duncker, K. (1945). On problem solving (L. S. Lees, Trans.). Psychological Monographs, 58(5, Whole No. 270). (Original work published 1935)

GeLmaN, R. (1990). Structural constraints on cognitive development. Cognitive Science, 14, 3-9.

Gemelli, A., \& CAPPellini, A. (1958). The influence of the subject's attitude in perception. Acta Psychologica, 14, 12-23.

Gibson, J. J. (1967). James J. Gibson. In E. G. Boring \& G. Lindzey (Eds.), A history of psychology in autobiography (pp. 125-143). New York: Appleton-Century-Crofts.

Gordon, I. E., DAy, R. H., \& STECher, E. J. (1990). Perceived causality occurs with stroboscopic movement of one or both stimulus elements. Perception, 19, 17-20.

Gruber, H. E., FinK, C. D., \& DAMM, V. (1957). Effects of experience on the perception of causality. Journal of Experimental Psychology, $53,89-93$.

Hume, D. (1978). A treatise of human nature. Oxford: Oxford University Press, Clarendon Press. (Original work published 1739)

Johansson, G., von HofsTen, C., \& JANSson, G. (1980). Event perception. Annual Review of Psychology, 31, 27-63.

JoYNSON, R. B. (1971). Michotte's experimental methods. British Journal of Psychology, 62, 293-302.

Leslie, A. M. (1982). The perception of causality in infants. Percep tion, 11, 173-186.

LESLIE, A. M. (1984). Spatiotemporal continuity and the perception of causality in infants. Perception, 13, 287-305.

LESLIE, A. M. (1988). The necessity of illusion: Perception and thought in infancy. In L. Weiskrantz (Ed.), Thought without language (pp. 185210). Oxford: Oxford University Press.

Leslie, A. M., \& KeEBle, S. (1987). Do six-month-old infants perceive causality? Cognition, 25, 265-288.

Michotте, A. (1963). The perception of causality (T. R. Miles \& E. Miles, Trans). London: Methuen. (Original work published 1946) MichotTe, A., \& Thinès, G. (1991). Perceived causality. In G. Thinès, A. Costall, \& G. Butterworth (Eds. \& Trans.) Michotte's phenomenology of perception (pp. 66-87). Hillsdale, NJ: Erlbaum. (Original work published 1963)

Millar, D. B. (1977). Perceptual cohesion in simple motion configurations. Unpublished doctoral dissertation, University of California, San Diego, La Jolla.

Natsoulas, T. (1961). Principles of momentum and kinetic energy in the perception of causality. American Journal of Psychology, 74, 394-402.

OAKes, L. M., \& CoHEN, L. B. (1990). Infant perception of a causal event. Cognitive Development, 5, 193-207.

Powesland, P. F. (1959). The effect of practice upon the perception of causality. Canadian Joumal of Psychology, 13, 155-168.

RuNeson, S. (1977). On visual perception of dynamic events. Unpublished doctoral dissertation, University of Uppsala, Uppsala, Sweden.

SCHLotTMANN, A. (1987). Judgments of causality in the perception of launch events. Unpublished master's thesis. University of California, San Diego, La Jolla.

Schlottmann, A., \& Shanks, D. R. (1992). Evidence for a distinction between judged and perceived causality. Quarterly Journal of Experimental Psychology, 44A, 321-342.

SHaNks, D. R. (1993). Human instrumental learning: A critical review of data and theory. British Journal of Psychology, 84, 319-354.

Thinès, G., Costall, A., \& Butterworth, G. (Eds. \& Trans.) (1991). Michotte's phenomenology of perception. Hillsdale, NJ: Erlbaum. 
UNDERWOOD, B. J. (1975). Individual differences as a crucible in the ory construction. American Psychologist, 30, 128-134.

WASSERman, E. A. (1990). Detecting response-outcome relations: Towards an understanding of the causal texture of the environment. Psychology of Learning \& Motivation, 26, 27-82.

WEIR, S. (1978). The perception of motion: Michotte revisited. Perception, 7, 247-260.

White, P. A. (1988). Causal processing: Origins and development. Psychological Bulletin, 104, 36-52.

ZALINSKI, J., \& ANDERSON, N. H. (1986). AVERAGE, a user-friendly FORTRAN-77 program for parameter estimation for the averaging model of information integration theory. La Jolla, CA: University of California, San Diego, Department of Psychology.

ZALINSKI, J., \& ANDERSON, N. H. (1991). Parameter estimation for averaging theory. In N. H. Anderson (Ed.), Contributions to information integration theory: Vol. I. Cognition (pp. 353-394). Hillsdale, NJ: Erlbaum.

\section{APPENDIX}

Because the interpretation of the data centered on the fivegroup classification of Figure 3 and the model-based parameters, the standard statistical analyses had secondary interest. Thus, the two instruction conditions produced different usages of the informational cues, as was seen in Figure 2, but an understanding of the usage depended on overriding this experimental variable with the individual differences classification. Some statistical details that may be of interest are noted here.

\section{Analysis for Two Instruction Conditions}

The overall analysis yielded significant main effects for gap, delay, and speed ratio $[F(3,144)=49.50,220.21$, and 10.41 , respectively]. (Results are reported at $p<.05$.) Only the gap effect differed in the two instruction conditions $[F(3,144)=$ 15.71]. Interactions of gap $\times$ delay, gap $\times$ speed ratio, and delay $\times$ speed ratio were found as well $[F(9,432)=17.68,3.72$, and 12.60 , respectively]. The interactions of both gap $\times$ delay and speed ratio $\times$ delay differed in the two instruction conditions $[F(9,432)=7.57$ and 2.82 , respectively]. These interactions reflect the difference in group patterns seen in Figure 2.

This overall analysis was a $2 \times 3 \times 2 \times 4 \times 4 \times 4$ mixed design analysis of variance (ANOVA) conducted on all 60 subjects' judgment data, with gender, type of prior subdesign, and instruction as between-subjects factors, and gap, delay, and speed ratio as within-subjects factors. Gender and type of subdesign entered into only one five-way interaction of marginal significance. Since there were 48 effects involving these two variables, this one significant effect may be a Type I error. Accordingly, these factors were not considered further.
Analyses for Individual Difference Groups

A 4 (gap) $\times 4$ (delay) $\times 4$ (speed ratio) ANOVA was run for each subject, with $320 d f$ for error. The means for the speed ratio variable were used to classify individuals into groups, as already noted. All but 2 subjects showed significant effects of delay, and all subjects except those in Group 1 showed significant effects of speed ratio. Twelve of 13 subjects in Group 1 showed significant effects of gap, whereas 21 of the remaining 47 subjects showed nonsignificant effects of gap, scattered through the remaining four groups with a somewhat higher frequency in Group 5. Roughly half of the subjects showed significant gap $x$ delay and speed ratio $x$ delay interactions, but only 10 of the 60 showed speed $\times$ gap interactions; only 5 showed three-way interactions. The majority of the gap $\times$ delay interactions - 19 of 29-were found in the causality instruction condition; the majority of the speed $\times$ delay interactions -22 of 36-were found in the naturalness instruction condition. These interactions reflect nonparallelism in the individual factorial graphs, which has been interpreted in terms of the weighting strategies associated with the averaging model.

Subjects were classified according to the speed ratio considered most causal/natural, which resulted in the five groups of Figure 3. A repeated-measures ANOVA was run for each group of Figure 3, and these are presented in Table A1. These $F$ ratios generally agree with the visual inspection of Figure 3 . The gap $\times$ delay interaction is significant for Groups $1-3$, which reflects the taper patterns visible in the left column of Figure 3. The gap $\times$ speed interaction is nonsignificant for all groups, which reflects the near parallelism in the center columns of Figure 3 . The delay $\times$ speed interaction is significant for all groups, which reflects the taper patterns in the right column of Figure 3, except for Group 1, in which the interaction arises from a small speed effect at no delay for some subjects.

This five-group classification naturally includes some individuals who did not fit exactly to the group pattern. Future researchers may find it useful to go into individual analysis in more detail. The usefulness of individual analysis, however, depends heavily on the applicability of the averaging model, which accordingly needs to be studied more extensively.

\section{Parameter Estimation}

The averaging model with differential weighting was fitted to the mean data for each of the five groups by applying the AVERAGE program (Zalinski \& Anderson, 1986, 1991) to the three-factor design. The program estimates 12 weights and 12 scale values, 1 each for each stimulus level and the initial expectation. The model requires the weights to sum to unity, so

Table A1

Summary Analyses of Variance: $\boldsymbol{F}$ Ratios

\begin{tabular}{|c|c|c|c|c|c|c|c|c|c|c|}
\hline \multirow[b]{3}{*}{ Source } & \multicolumn{10}{|c|}{ Group } \\
\hline & \multicolumn{2}{|c|}{1} & \multicolumn{2}{|c|}{2} & \multicolumn{2}{|c|}{3} & \multicolumn{2}{|c|}{4} & \multicolumn{2}{|c|}{5} \\
\hline & $F$ & $M S_{e}$ & $F$ & $M S_{e}$ & $F$ & $M S_{\mathrm{e}}$ & $F$ & $M S_{e}$ & $F$ & $M S_{e}$ \\
\hline Gap & $42.57^{*}$ & 14,176 & $10.06 *$ & 14,993 & $6.75^{*}$ & 16,111 & 1.34 & 4,117 & $5.58 *$ & 3,596 \\
\hline Delay & $19.78^{*}$ & 12,005 & $72.08 *$ & 7,502 & $27.86^{*}$ & 10,899 & $34.51^{*}$ & 9,475 & $179.45^{*}$ & 5,612 \\
\hline Speed Ratio & 0.62 & 835 & $56.96 *$ & 6,837 & $20.45^{*}$ & 7,174 & $8.84 *$ & 7,478 & $26.30^{*}$ & 6,395 \\
\hline Gap $\times$ delay & $10.67 *$ & 2,092 & $7.99 *$ & 1,141 & $5.44 *$ & 1,718 & 1.32 & 584 & 1.29 & 709 \\
\hline Gap $\times$ speed ratio & 1.43 & 873 & 1.10 & 619 & 1.03 & 486 & 1.04 & 426 & 1.32 & 469 \\
\hline Delay $\times$ speed ratio & $3.64^{*}$ & 713 & $10.06^{*}$ & 866 & $11.36^{*}$ & 1,001 & $3.04 *$ & 1,227 & $11.91 *$ & 972 \\
\hline Gap $\times$ delay $\times$ speed ratio & 1.25 & 588 & 1.06 & 357 & 0.87 & 656 & 1.09 & 504 & 1.09 & 464 \\
\hline
\end{tabular}

Note-MS $\mathrm{s}$ are for scores on 300-point scale; numerator $d f \mathrm{~s}$ are 3, 9, and 27 for main effects, two-way, and three-way interactions, respectively; denominator $d f$ s depend on group size listed in Table $1 .{ }^{*} p<.05$. 
there are only 23 free parameters. This was reduced to 19 for Groups 1,4 , and 5, as noted in the text. There were 48 data points, so the data:parameter ratio was slightly above $2: 1$. With this ratio, the AVERAGE program yields stable estimates with no problems of local minima, as shown in Monte Carlo studies by Zalinski and Anderson (1991).

If the full design could have been supplemented with subdesigns that involved only one or two of the three cues, the model could yield weight estimates for all three variables on a common ratio scale. Such subdesigns, unfortunately, may not be realizable in the present task; all three variables are physically inherent in the launch event. A launch event always contains, for example, some spatial contiguity information. The level of uniqueness of the weight estimates, accordingly, may be no more than a linear scale for each separate variable. This allows comparisons of differences between weight estimates within each dimension, but comparisons across dimensions are problemati$\mathrm{cal}$. Scale values suffer an associated bias, although the meaningfulness of the estimates in Table 2 suggests that this bias was small.
The initial state variable, which may be conceptualized as an expectancy for the average launch event, yielded estimates of $\psi_{0}$ close to the neutral midpoint of 150 for each group and of $w_{0}$ around -5 for each group. This negative estimate is not an anomaly. Similar negative values were obtained with Monte Carlo runs on perfect data generated from the averaging model, and reflect the indicated level of uniqueness. It results from the program, which leaves $w_{0}$ unbounded in the estimation.

In future work, it may be useful to seek some additional variable that is not physically integral to the perception of the launch event, one that will be ignored unless it is specifically made salient. Relative size, for example, would presumably have an effect if manipulated over more than one level, but perhaps not if held constant at one value. If such variables can be established, they may provide a useful sharpening of uniqueness for the weight estimates. The AVERAGE program can be used to test for uniqueness properties of a design before it is run.

(Manuscript received September 17, 1992; revision accepted for publication May 3, 1993.) 